\title{
Semi-alicyclic polyimides as potential membrane oxygenators: Rheological implications on film processing, morphology and blood compatibility
}

\author{
R. M. Albu, C. Hulubei, I. Stoica, A. I. Barzic* \\ 'Petru Poni’ Institute of Macromolecular Chemistry, 41A Grigore Ghica Voda Alley, 700487 Iasi, Romania
}

Received 10 September 2018; accepted in revised form 21 November 2018

\begin{abstract}
Two polyimide structures were prepared from an alicyclic dianhydride and aromatic diamines, containing or lacking fluorine, and were tested as potential membrane oxygenators. First, the solution rheological properties were investigated to evaluate their effect on film processing by tape casting. Velocity and shear rates profiles were simulated to determine the shearing conditions during tape casting and transfer them in the flow curves. Proper films were obtained at a viscosity corresponding to a concentration in entangled regime, where pseudoplastic behavior is still noticed at shear rates beneath the blade. Theoretical predictions of gas permeability indicated a good oxygen $\left(\mathrm{O}_{2}\right)$ exchange and carbon dioxide $\left(\mathrm{CO}_{2}\right)$ removal through the studied polymers. Atomic force microscopy (AFM) scans revealed a morphology characterized by an intrinsic porosity. The pore size, uniformity and size distribution was affected by the structure of the diamine moiety. The interactions of blood with polyimide films indicated no prevalent adhesion of cells or plasma proteins. The results showed that the examined samples have adequate properties for potential membrane oxygenator applications.
\end{abstract}

Keywords: material testing, rheology, polyimide, hemocompatibility

\section{Introduction}

Current approaches in medicine are based on a wide range of synthetic and hybrid bio-compounds [1]. Among them, polymeric biomaterials have drawn a particular attention due to the similarities with tissues and the ease of processability into elements for medical devices [2]. In some situations, the biomaterial must be brought in contact with blood permanently or transiently upon implantation [3]. The contact between blood and non-biological materials determines the activation of the cascade systems, which generates activation of leukocytes and thrombocytes [4]. Although significant progress concerning hemocompatibility has been made, certain materials are still rendering undesired side effects. Therefore, many efforts have been devoted to establish the ideal material characteristics in terms of platelet activation, blood coagulation ability or bacterial attachment. Literature survey on this subject indicates that there are several types of polymer structures that could fulfill the basic requirements for hemocompatibility, such as poly(ethylene terephthalate) [5], polyacrylonitrile [6], modified cellulose [7], polydimethylsiloxane [8], and polyurethane [9]. In the past years, another class of polymers emerged as bio- and hemocompatible materials, namely polyimides (PIs) [10]. They have imide rings along the backbone, which confer high thermo-oxidative resistance, enhanced mechanical strength and superior chemical resistance [11]. Despite their promising properties for biomedicine, little has been published regarding PI compatibility with cells/tissues and blood. Haggerty and Lusted [12] studied the reactions of cochlea tissue with PI-based implants. Seo 
et al. [13] investigated in vitro the biocompatibility of a flexible polyimide microelectrode array with respect to human retinal pigment epithelial cells for retinal stimulation purposes. In vivo tests indicated good stability and no harmful effects in rabbit eyes by 12 weeks [13]. The minimal tissue reactions and reduced inflammation close to the implant region were noticed. The problem of PI hemocompatibility was mainly analyzed by of Kawakami and coworkers [14-17], which proved that no significant thrombus is formed on the polymer surface after in vivo testing. Combining this bio-feature with gas separation properties, Kawakami [14-17] developed novel membrane oxygenators. All these reports are focused on fully aromatic PIs of commercial nature [12] or synthesized from monomers containing fluorine and/or sulfone groups [14-17]. Since 2012, our research group initiated the studies on hemocompatibility of PI based on aliphatic sequences [18-22]. A particular attention was ascribed to PIs prepared from 5-(2,5-dioxotetrahydrofuryl)-3-methyl-3-cyclohexene-1,2-dicarboxylic acid anhydride (EPI), which was proven to have good biocompatibility with dental or dermal tissues [23, 24].

Tape casting (or knife coating) is one of the most widespread techniques for fabrication of thin films on large area surfaces, with thickness ranging from $25 \mu \mathrm{m}$ up to $1 \mathrm{~mm}$ [25]. The underlying principle of this method is that a reservoir of solution with a slit shaped outlet leaves a layer of solution. Depending on the polymer behavior in solution and the speed of blade movement, one may control the thickness of the tape. Therefore, the rheological properties of the polymer solution are essential for establishing the optimal conditions in which the film can be produced. For instance, a reduced yield stress or its absence for polymer solution is desirable if low shear stress (small casting velocity, low viscosity, a big blade gap and/or a low amount of solution in reservoir) is the driving force of the casting process. Viscosity variation with applied shear underneath the moving blade is responsible for the tape leveling and for solution flowing aside, which could affect the shape of the tape [25].

In the presented scientific context, this work brings new aspects concerning the processing and characterization of semi-aliphatic PIs for membrane oxygenator purposes. Two semi-alicyclic PI structures were prepared by polycondensation reaction using a similar procedure to that reported in other works $[26$,
27]. For PI synthesis, EPI dianhydride was combined with two aromatic diamines (containing or lacking fluorine): 4,4'diaminodiphenylmethane (DDM) and 4,4'-(hexafluoroisopropylidene)bis(p-phenyleneoxy) dianiline (6FADE). The European Commission for 'Employment, Social Affairs \& Inclusion SCOEL' warns about the DDM toxic and carcinogenic nature. However, we used this monomer in the polymer chemistry taking into account the exceptional stability of a PI type structure from the chemical, thermal, UV and the high tolerance against radiations. Replacement of DDM monomer in the polymerization of monomer reactants of the PI with a monomer containing hexafluoroisopropylidene bridge has provided us a PI alternative structure aiming: (1) eliminating potential toxicity and carcinogenic aspects of the initial polymer, reducing in this way the health effects associated with the DDM monomer in various possible applications, as well as those relating to environmental protection; (2) conservation of the superior thermal and mechanical properties of a PI type polymer; (3) development of new structure-properties relationships that will enable similar or improved performance relative to the reference one.

In a previous paper, these polymers were studied from the point of view of their optical properties implication on the possibility to reduce losses in display devices [27]. Here, a special concern is ascribed to the correlation of the rheological behavior of PI solution in order to obtain proper films by tape casting. As far as we know, such study has not yet been reported. The EPI dianhydride is expected to render biocompatibility to the corresponding PIs, while its non-symmetric and bulky character combined with diamine moiety peculiarities is expected to generate an intrinsic porosity to the resulting PI films/membranes. The hemocompatibility was tested by directly placing the biological liquid on PI supports and then after a considerable period of interaction, the adhesion/cohesion of blood components at bio-interface was evaluated. To our knowledge, this combination of procedures for evaluation of blood compatibility of PIs has not been reported.

\section{Experimental}

\subsection{Materials and PI synthesis}

The monomer 5-(2,5-dioxotetrahydrofuryl)-3-methyl3-cyclohexene-1,2-dicarboxylic acid anhydride (EPI) was purchased from Merck Chemical Co (Germany). The reactants 4,4'diaminodiphenylmethane (DDM), 


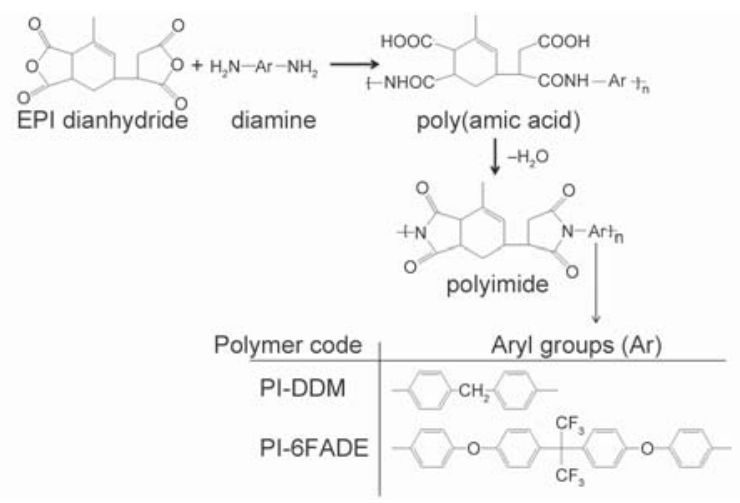

Figure 1. The synthesis steps of the analyzed semi-alicyclic polyimides.

4,4'-(hexafluoroisopropylidene)bis(p-phenyleneoxy) dianiline (6FADE) and the solvent N-methylpyrrolidinone (NMP, 99.5\% anhydrous) were achieved from Sigma-Aldrich (Germany). The analyzed semialicyclic PIs were obtained by the polycondensation reaction between EPI dianhydride with either the DDM or 6FADE diamines in NMP medium (see Figure 1).

The details concerning the synthesis procedure were described in a previous work [27]. Fourier transformed infrared spectra, previously published in [27], confirmed the studied polymer structures.

\subsection{Molecular weight and solubility}

The molecular weights of the PI samples were determined by gel permeation chromatography (GPC) using a PL EMD-950 evaporative mass detector (Polymer Laboratories Ltd, UK). Polystyrene standards were used as calibration standards. The number average molecular weight $\left(M_{\mathrm{n}}\right)$ values and polydispersity indices (PDI) are given in Table 1.

The solubility of the examined PIs is also presented in Table 1. Both polymers are soluble in polar organic solvents, namely $N$-methylpyrrolidinone (NMP), dimethylacetamide (DMAc), dimethylformamide (DMF), and dimethyl sulfoxide (DMSO). They are insoluble in weak polar or non-polar solvents, excepting $m$-Cresol in the case of poly(EPI-DDM) and $m$-Cresol, acetone, tetrahydrofuran (THF) and chloroform $\left(\mathrm{CHCl}_{3}\right)$ in the case of poly(EPI-6FADE).
Compared with the Kapton PI (which is insoluble in all the studied organic solvents) the discussed PIs especially poly(EPI-6FADE) - have an improved solubility induced by a synergistic effect between the greater freedom of sequences movement $\left(-\mathrm{C}\left(\mathrm{CF}_{3}\right)_{2}-\right.$ or $-\mathrm{CH}_{2}-$ ), which decrease the entropy of internal rotation, and the presence of the bulky non-symmetric cycloaliphatic EPI structure, respectively; this set of factors reduces inter- and intramolecular interactions allowing the migration of solvent molecules into the polymer mass.

\subsection{Solution and film preparation}

The PI solutions in NMP were prepared based on a simple procedure. Various amounts of solid samples were weighted and placed in glass flasks to achieve the concentrations of 3, 7, 12, 17, 23 and $28 \mathrm{~g} / \mathrm{dl}$. After the solubilization of the PI, the resulting systems were deposited on substrates by tape casting method. The procedure was done at a casting gap of 1 and at a constant speed $(1.5-18.8 \mathrm{~mm} / \mathrm{s})$. The samples were dried in a vacuum oven using a heating program, where temperatures ranged between 80 and $220^{\circ} \mathrm{C}$. The density in solution is 1.02 and $1.21 \mathrm{~g} / \mathrm{cm}^{3}$ and solid state is 1.49 and $1.63 \mathrm{~g} / \mathrm{cm}^{3}$ for PI-DDM and PI-6FADE samples, respectively.

\subsection{Molecular modeling}

The molecular modeling was made with HyperChem software (Demo version). Initially, the 3D structures of each semi-aliphatic PI were subjected to a geometry optimization procedure by applying AMBER force field and Polak-Ribiere algorithm. The PI conformation with the lowest energy was then used to simulate the conformational changes in the presence of seven NMP molecules.

\subsection{Rheology}

The rheological characteristics of the PI solutions in NMP were determined on a Bohlin CS50 device (Malvern Instruments, UK), which was equipped with cone/plate system $\left(4^{\circ} / 40 \mathrm{~mm}\right)$. The flow curves were obtained over the $0.02-500 \mathrm{~s}^{-1}$ shear rate interval.

Table 1. Molecular weight and solubility properties of the studied PIs.

\begin{tabular}{|c|c|c|c|c|c|c|c|c|c|c|}
\hline \multirow{2}{*}{ PI code } & \multirow{2}{*}{$\begin{array}{c}M_{\mathrm{n}} \\
{[\mathrm{g} / \mathrm{mol}]}\end{array}$} & \multirow{2}{*}{ PDI } & \multicolumn{8}{|c|}{ Polymer solubility } \\
\hline & & & NMP & DMAc & DMF & DMSO & $m$-Cresol & Acetone & THF & $\mathrm{CHCl}_{3}$ \\
\hline poly(EPI-DDM) & 21481 & 1.60 & ++ & ++ & ++ & ++ & ++ & - & - & - \\
\hline poly(EPI-6FADE) & 23900 & 1.81 & ++ & ++ & ++ & ++ & ++ & +- & + & +- \\
\hline
\end{tabular}

where $++=$ good solubility, $+-=$ partial solubility, $-=$ insolubility 
All experiments were performed at the temperature of $25^{\circ} \mathrm{C}$.

\subsection{Contact angle}

The contact angles were determined using a labmade device equipped with a Hamilton syringe. The interactions occurring at polymer/blood interface were assessed by measuring the contact angle of blood drops on the samples.

\subsection{Morphology}

The surface morphology of the semi-alicyclic PI films was characterized by means of atomic force microscopy (AFM) on SPM SOLVER Pro-M instrument (NT-MDT, Zelenograd, Russia), in air at $23^{\circ} \mathrm{C}$, in tapping mode, using a rectangular cantilever NSG10/Au (NT-MDT, Zelenograd, Russia) with a resonant frequency of $213 \mathrm{kHz}$. The $2.5 \times 2.5 \mu \mathrm{m}^{2}$ scanning areas were analyzed for each sample by means of Nova v.1.26.0.1443 for Solver software. The parameters depicting the surface features were calculated using Image Analysis 3.5.0.18542 software. The pore maps and diagrams of the pore diameter /aspect ratio distribution were also obtained using the latter software.

\subsection{Thermal properties}

The thermogravimetric experiments were done on a Mettler Toledo TGA/SDTA 851 derivatograph (Mettler Toledo, Switzerland). The thermogavimetric (TG) and derivative thermogravimetric (DTG) curves were obtained in inert (nitrogen) atmosphere in the temperature interval of $25-700^{\circ} \mathrm{C}$, using a heating rate of $10^{\circ} \mathrm{C} / \mathrm{min}$.

\subsection{Tensile properties}

The tensile properties of PI films were measured on a Shimadzu AGS-J deformation device (Shimadzu, Japan) at ambient temperature.

\subsection{Blood cell/PI interactions}

The interactions of the blood cells with PI samples was evaluated using $0.5 \mathrm{ml}$ of biological fluid (3.8\% anticoagulant). The biological fluid was voluntary provided by a healthy human donor and was left to interact with $1 \mathrm{~cm}^{2}$ polymer surface area for $4 \mathrm{~h}$ at $37^{\circ} \mathrm{C}$. Subsequently, the specimens were washed three times with Phosphate Buffer Saline (PBS, Sigma-Aldrich, Germany) and rinsed with distilled water. After drying, the samples were analyzed on the optical microscope to monitor the blood cell attachment.

\section{Results and discussion}

\subsection{Molecular simulations analysis}

Molecular simulation represents a powerful tool for analysis of the polymer conformation in vacuum or other media. The thermodynamic quality of the solvent affects the polymer conformation in solution and consequently in the corresponding film. In a good solvent, the chains tend to interpenetrate more easily, leading to a film with a denser structure [28]. For this reason, NMP was selected as the working solvent.

The shape of the structural unit (SU) corresponding to the optimized PIs was simulated in a cage containing seven molecules of NMP solvent. As seen in Figure 2, the macromolecular conformation is impacted by the structure of both dianhydride and diamine segments. A better differentiation can be done by examining first the effects of the common part of each PI structure, namely the alicyclic dianhydride, and then those generated by the aromatic diamine. The EPI moiety contains a single bond connecting the imide group and alicyclic ring, which produces a bond angle of 112.0 and $111.4^{\circ}$ for PI-DDM and PI-6FADE, respectively (see Figure $2 \mathrm{a}$ and $2 \mathrm{~b}$ ).

The length and the number of kinks in the diamine moiety clearly determine a distinct spatial arrangement of PI chains around the solvent molecules. The sample containing short DDM unit has a lower degree of freedom for rotation around single bonds. The DDM sequence presents a main point of chain folding around the methylene group, with a bond angle of $114.8^{\circ}$. In the case of PI-6FADE, the presence of the ether bridges and 6FADE group, intercalated between the phenyl rings, introduces an enhanced degree of freedom for rotation around single bonds. So, the 6FADE unit is characterized by three points of chain bending, with different bond angles: $105.2^{\circ}$ for carbon atoms around $6 \mathrm{~F}$, while in the zone of ether groups the bond angles are 127.6 and $125.8^{\circ}$ (see Figure 2b). The differences induced by the stereochemical peculiarities of the two types of the structural units were discussed based on the macromolecular conformation of the corresponding PIs and were schematically illustrated under the form of red lines. As noticed in Figure 2a and 2b, the SU of PIDDM can be viewed as being almost similar with the ' $u$ ' letter owing to its two points of chain folding, whereas the SU of PI-6FADE presents four points 
a)

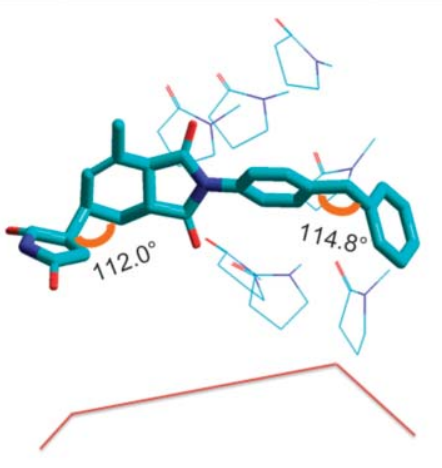

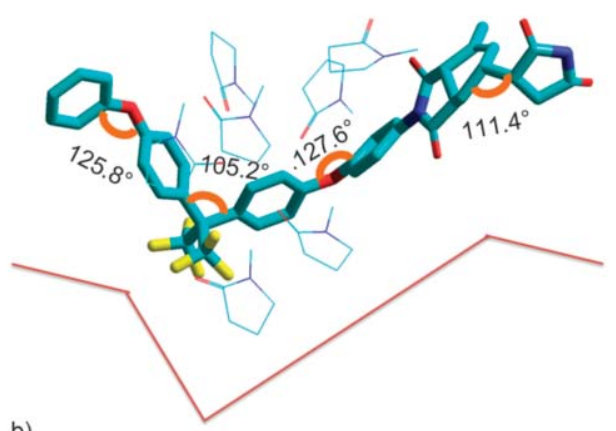

b)

Conformation of two interacting $\mathrm{PI}$ chains of $30 \mathrm{SUs}$

c)

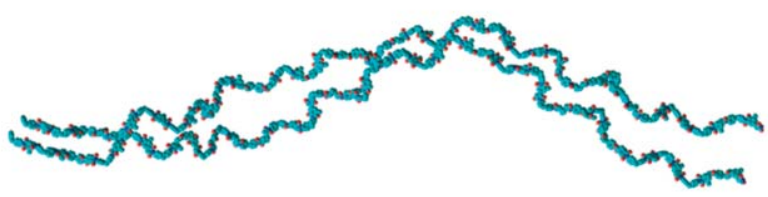

d)

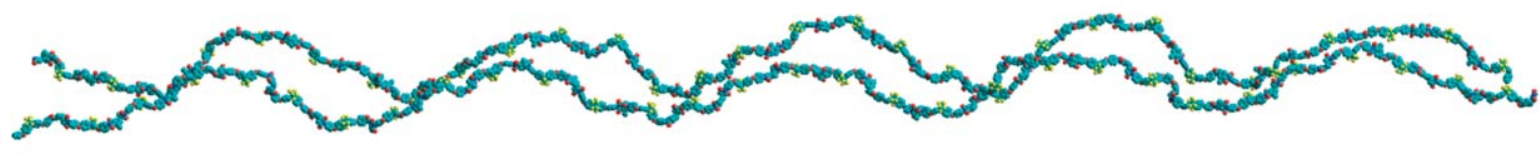

Figure 2. Molecular modeling in a cage of seven molecules of NMP interacting with the SU of: (a) PI-DDM and (b) PI6FADE (the red lines represent the overall shape of PI chains). The spatial molecular conformation of two interacting PI chains containing 30 SUs for (c) PI-DDM and (d) PI-6FADE.

where the chain bends. This observation may be connected to greater flexibility of the polymer chain PI6FADE versus PI-DDM; the glass transition temperature is higher for the PI-DDM $\left(241^{\circ} \mathrm{C}\right)$ than PI6FADE $\left(215^{\circ} \mathrm{C}\right)$, confirming this association.

In order to see how the SU configuration affects the PI conformation at macromolecular level, we simulated the shape of two interacting PI chains containing 30 SUs. The corresponding spatial molecular conformations are depicted in Figure $2 \mathrm{c}$ and $2 \mathrm{~d}$. It can be noticed that the macromolecule that contains 6FADE segments presents more bending points along the backbone, which confirm again its higher chain flexibility comparatively with PI-DDM.

\subsection{Rheological effects on PI processing}

The viscosity $(\eta)$ is an essential factor in understanding the structure-function relation of macromolecular materials. This parameter it is closely linked to the micro-structural changes in different conditions, e.g. shear field, concentration, temperature, etc. The studied PI solutions in NMP present a shear rate dependent viscosity starting with a critical shear rate $\left(\dot{\gamma}_{\mathrm{c}}\right)$, denoting the pseudoplastic character (see Figure 3).
The region from the flow curves, where the viscosity is independent of the applied shear field, marks the Newtonian behavior. The transition from the shear rate independent to the shear rate dependent viscosity is influenced by the PI structure. To clarify the differences in the rheological properties, a deep examination of the influence of PI microstructure on viscosity must be made. In the absence of the shear field (static conditions), the macromolecules intertwine and entangle with each other in manner dependent on the PI molecular weight. As the chain length is higher, more entanglements are formed. Given the fact that entanglements resist flow, under near static conditions, the resistance to flow is at its highest level and here the zero-shear viscosity can be extracted.

When the PI solutions have the lowest concentration (i.e. $3 \mathrm{~g} / \mathrm{dl}$ ) the degree of chain entanglements is smaller. As these samples are subjected to increasing shear, the chains begin to slide over each other and gradually orient along the flow direction. So, the studied systems display a progressive reduction of the viscosity. In this shearing interval (below $1 \mathrm{~s}^{-1}$ ), the viscosity of PI-DDM sample is slightly lower, 

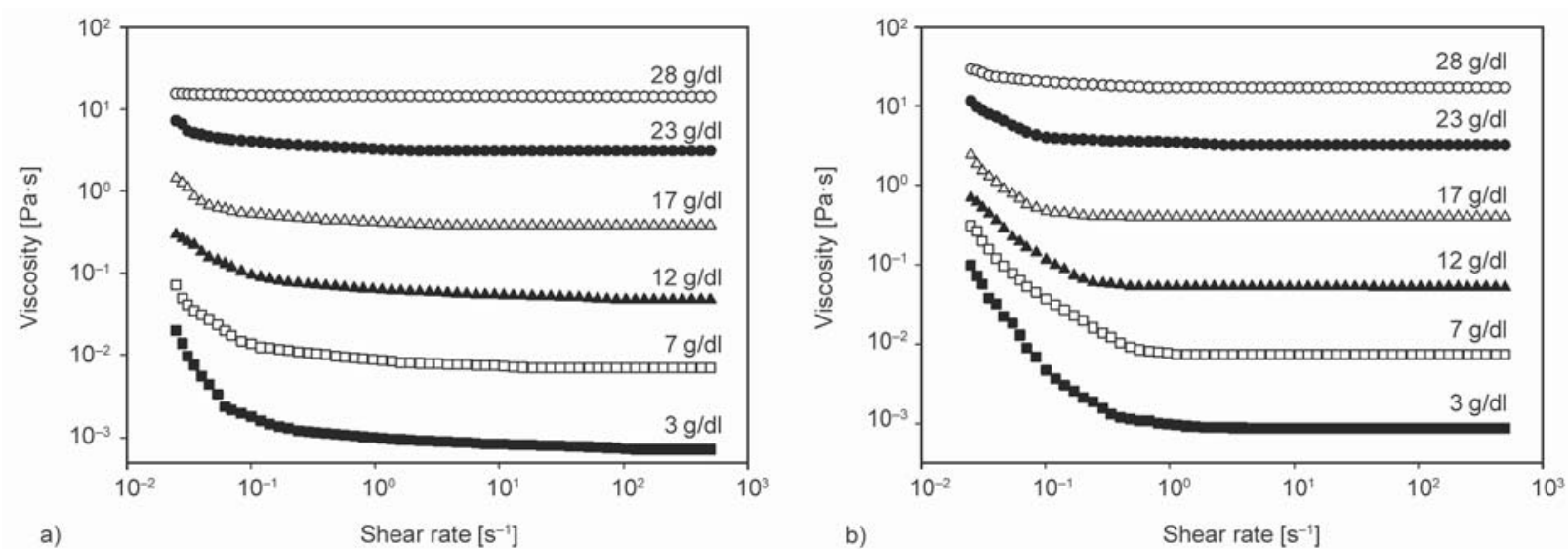

Figure 3. Viscosity variation with shear rate for (a) PI-DDM and (b) PI-6FADE solutions.

compared to that of the PI-6FADE, owing to its smaller molecular weight. Moreover, the DDM diamine segment is shorter and more rigid, determining less steep viscosity curves. Conversely, the 6FADE units are longer and the combination of ether linkages with $6 \mathrm{~F}$ bridge allow faster disentanglements at low shear rates, thus the pseudoplastic behavior is more pronounced and is expanded to a higher shear domain in comparison with PI-DDM sample. After the gradual loss of entanglements, as the shearing is further enhanced the PI chains begin to align along the flow direction and the viscosity tends to be independent of applied shear rate. The higher backbone flexibility and molecular weight of PI-6FADE enable denser entanglements so the magnitude of viscosity is slightly high even at high shear rates. As the PI concentration in solution rises, the viscosity increases at low shear rates and to a limited extent at high shearing because the intermolecular interactions are restricted. The effect observed at small shear rates is depicted more comprehensibly in the plot of specific viscosity $\left(\eta_{\mathrm{sp}}\right)$ against concentration (c) on a log-log scale (see Figure 4).

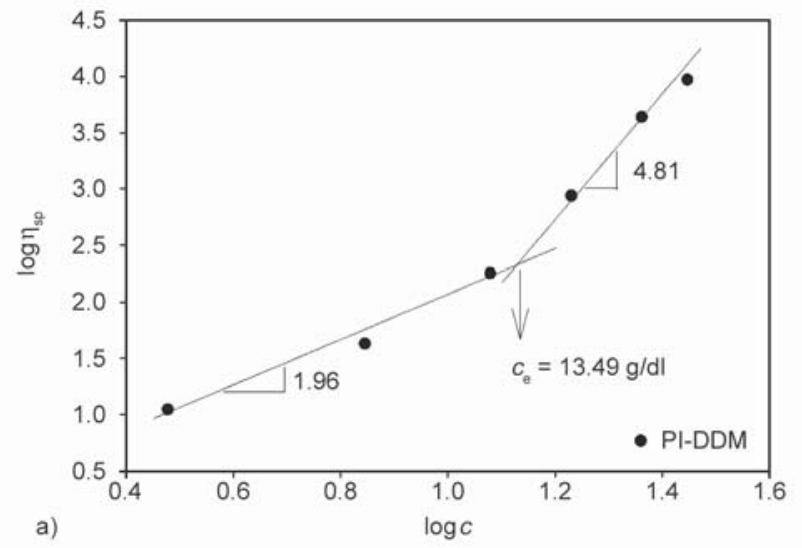

It can be discerned that $\eta_{\text {sp }}$ follows a power-law relationship with the solution concentration: $\eta_{\mathrm{sp}} \sim c^{\mathrm{x}}$. According to Krause et al. [29], the values of the exponent $x$ make the difference between the semidilute unentangled and the semidilute entangled regimes. For solutions of linear polymers, where the polymer chains interact prevalently with solvent molecules, the exponent is about 1.25 , whereas in the domain with dominant interchain entanglements, the exponent ranges in the interval 4.25-4.5. A similar $\eta_{\text {sp }}$ dependence on concentration was obtained for the studied PI solutions. Thus, in the concentration domain of 3-12 $\mathrm{g} / \mathrm{dl}$, the systems are in semidilute regime where chains are not overlapped, namely $x$ is 1.96 and 1.41 for PI-DDM and PI-6FADE, respectively. In the range of $17-28 \mathrm{~g} / \mathrm{dl}$, the samples are found in the semidilute entangled zone as the exponent $x$ suddenly increases up to 4.81 and 4.99 for PI-DDM and PI6FADE, respectively. The crossover point from the semidilute unentangled to semidilute entangled zone denotes the critical entanglement concentration $\left(c_{\mathrm{e}}\right)$. The difference between the values of this parameter can be explained by considering that a higher PI

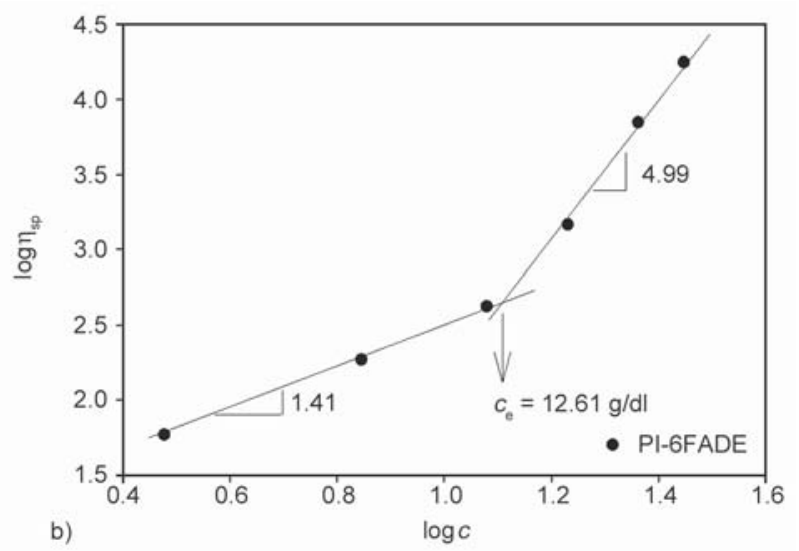

Figure 4. Specific viscosity against PI solution concentration for (a) PI-DDM and (b) PI-6FADE. 
molecular weight produces an increased number of entanglements and thus they appear at a lower concentration for PI-6FADE than for PI-DDM (see Figure 4).

For all analyzed systems, the value of the critical shear rate decrease with increasing the concentration from $3 \mathrm{~g} / \mathrm{dl}\left(\dot{\gamma}_{\mathrm{c}}=0.24-1.13 \mathrm{~s}^{-1}\right)$ to $28 \mathrm{~g} / \mathrm{dl}\left(\dot{\gamma}_{\mathrm{c}}=0.03\right.$ $\left.0.04 \mathrm{~s}^{-1}\right)$. The Newtonian region is extended when the sample concentration raises. In other words, all solutions have shorter psedudoplastic regions when more PI chains are present in solution. This could be explained by considering that further increase in concentration leads to more entangled coils, which are less able to comb out and orient along the flow direction. Thus, at the highest concentration of $28 \mathrm{~g} / \mathrm{dl}$, the viscosity becomes almost entirely independent of the applied shear, except for small deviations at very low shear rates. At this level of high concentration of entangled macromolecular coils in solution, the molecular network is stronger and is less possible to be disrupted.

For processing purposes, the concentration from semidilute entangled regime at which the pseudoplastic behavior is still pronounced was selected, namely $17 \mathrm{~g} / \mathrm{dl}$. The model proposed by Siko [30] was used to describe the non-Newtonian viscosity of the samples (Equation (1)):

$\eta=\eta_{\infty}+k \cdot \dot{\gamma}^{\mathrm{n}-1}$

where $\eta_{\infty}$ is the infinite shear rate viscosity, $n$ is the flow behavior index, $k$ is the consistency index.

This model is typical for shear thinning at low shear rates followed by a Newtonian plateau, as remarked for the analyzed PI solutions at the concentrations smaller than $28 \mathrm{~g} / \mathrm{dl}$. The parameters resulted after fitting the rheological data with Sisko model [30] at $17 \mathrm{~g} / \mathrm{dl}$ are displayed in Table 2.

The magnitude of $\eta_{\infty}$ ranges in agreement with the PI molecular weight. The value of $\mathrm{k}$ is related to the consistency of the PI solution. The PI-6FADE system exhibits slightly higher consistency comparatively with the PI-DDM solution due to the higher

Table 2. Summary of Sisko parameters for the studied PI solutions at the concentration of $17 \mathrm{~g} / \mathrm{dl}$.

\begin{tabular}{|l|c|c|c|}
\hline PI solution & $\begin{array}{c}\boldsymbol{\eta}_{\infty} \\
{[\mathbf{P a} \cdot \mathbf{s}]}\end{array}$ & $\begin{array}{c}\boldsymbol{k} \\
{\left[\mathbf{P a} \cdot \mathbf{s}^{\mathbf{n}}\right]}\end{array}$ & $\boldsymbol{n}$ \\
\hline PI-DDM & 0.37 & 0.03 & 0.31 \\
\hline PI-6FADE & 0.41 & 0.05 & 0.12 \\
\hline
\end{tabular}

concentration of chain entanglements in solution. The flow behavior index can be viewed as a measure of degree of thinning. For $n=1$, the rheological model is reduced to Newtonian behavior, where $\eta=\eta_{0}$. At the highest studied concentration of $28 \mathrm{~g} / \mathrm{dl}$ all PI solutions have a flow index close to unity. As the concentration decreases the pseudoplastic character becomes prevalent, thus the values of $\mathrm{n}$ are positive and sub-unitary $(n<1)$. As the flow index falls from unity, one may see that the shear thinning behavior becomes more pronounced. The flow properties at $17 \mathrm{~g} / \mathrm{dl}$ still preserve the shear thinning behavior. The flexible PI-6FADE chains lead to steeper viscosity curves in regard to the PI-DDM solution. Therefore, the latter PI has slightly higher flow index value.

The shear thinning behavior is very important in the processing stage of the PI into films by tape casting because the solution viscosity decreases when passes underneath the casting blade. This simplifies the uniform flow and overall leveling. Moreover, the viscosity is enhanced while the polymer solution is dragged from the casting unit, hindering the solution from flowing aside and preserving the film shape. Given the fact that the viscosity of the samples is shear stress dependent and determines modifications during the casting process, it is necessary to study the real flow profile inside the casting unit. The system of the driving forces acting during the flow is complex. The gradual reduction of the load in the tank makes the flow volume under the blade to be affected by the pressure-driven flow. In addition, the steady state carrier speed is another factor that affects the flow volume below the blade. Therefore, the wet film thickness is influenced by Poiseuille flow, shear rate, drag-driven flow and Couette flow. Jabbari et al. [31] developed a model for describing the shear rate and the pressure-driven flow in the casting unit, which included the dropping of the solution volume during film preparation. The local velocity profiles could be examined based on the analytical solution of Poiseuille-Couette flow depicted by Equation (2):

$v_{\mathrm{x}}=v \frac{z}{h}+h^{2} P \frac{z}{h} \cdot\left(1-\frac{z}{h}\right)$

where $h$ is the casting gap, $v$ is the draw velocity, $z$ is the position under the blade and $P$ is a factor that includes the effects of the driven-pressure flow in regard with the solution viscosity and blade size. 

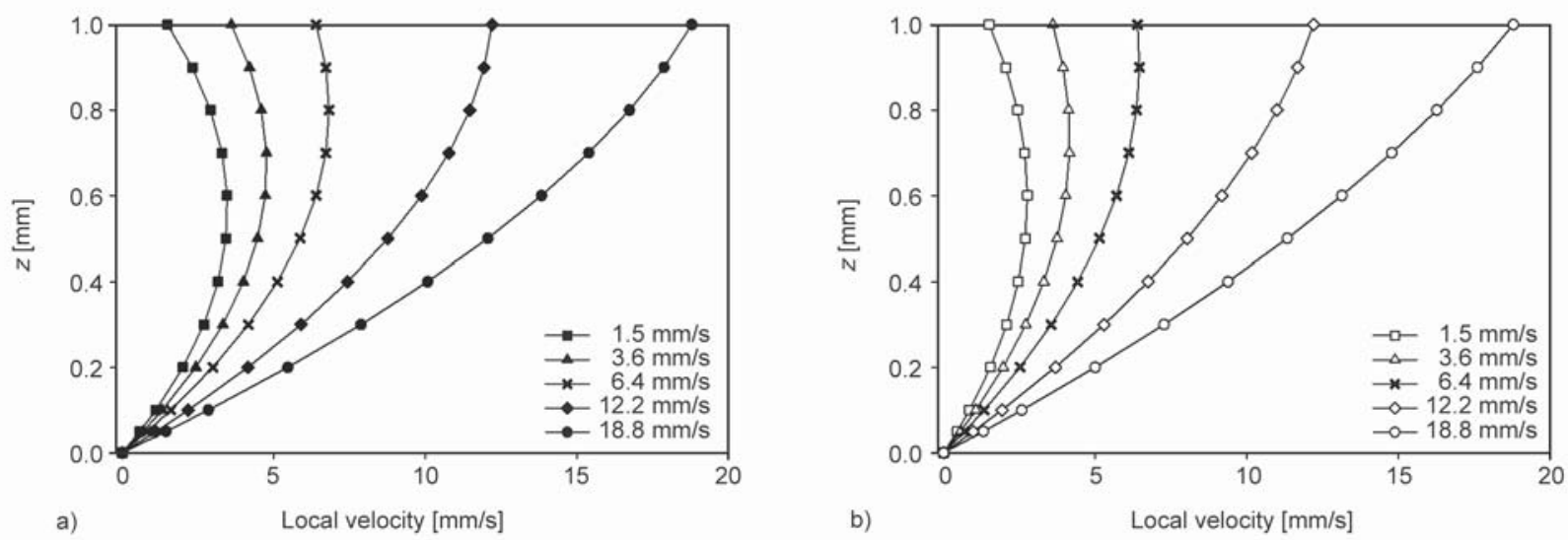

Figure 5. Velocity profiles underneath the casting blade as the function of the applied velocity of the carrier for (a) PI-DDM and (b) PI-6FADE solutions.

Using the rheological parameters from Figure 3 at $17 \mathrm{~g} / \mathrm{dl}$, the gap of $1 \mathrm{~mm}$ and casting speed in the interval of $1.5-18.8 \mathrm{~mm} / \mathrm{s}$ as input data, the velocity and shear rate profiles were obtained. Figure 5 illustrates the velocity profiles underneath the casting blade. It was noticed that as the draw velocity increases, the distribution changes its shape becoming almost linear. This situation corresponds to Couette flow conditions. For smaller velocities, the curves do not display a linear correlation because the hydrostatic pressure is prevailing. So, one may observe a peak, which arises from the combination of Poiseuille and Couette flow conditions.

The numerical simulations allowed determining the relevant working interval of shear rates beneath the casting blade. This is very important because a small variation of the velocity generates a wide scatter of the shear rates. The distributions of the shear rate across the gap at several draw velocities are depicted in Figure 6. It can be remarked that the biggest variation of the shear rates under the blade is in the range of $0-30 \mathrm{~s}^{-1}$ for PI-DDM solution and $0-26 \mathrm{~s}^{-1}$ for PI6FADE system. Transferring these shear rate ranges in the flow curves obtained by means of rheology, a rough analysis of how PI solution viscosity changes below the blade became possible. In this region of shear rates, the viscosity graphs are flat above $0.1 \mathrm{~s}^{-1}$, as seen in the insets from Figure 6. This study indicates that the PI solutions are characterized by the pseudoplastic behavior, necessary for tape casting. Also, the samples are stable during the casting process and the rheological properties of the PI systems can be described with a consistent viscosity for a whole tape casting process carried with $1 \mathrm{~mm}$ gap and $18.8 \mathrm{~mm} / \mathrm{s}$ speed.

To further understand how the rheological interfere in PI processing by solution tape casting, we monitored some factors that affect the PI film thickness.
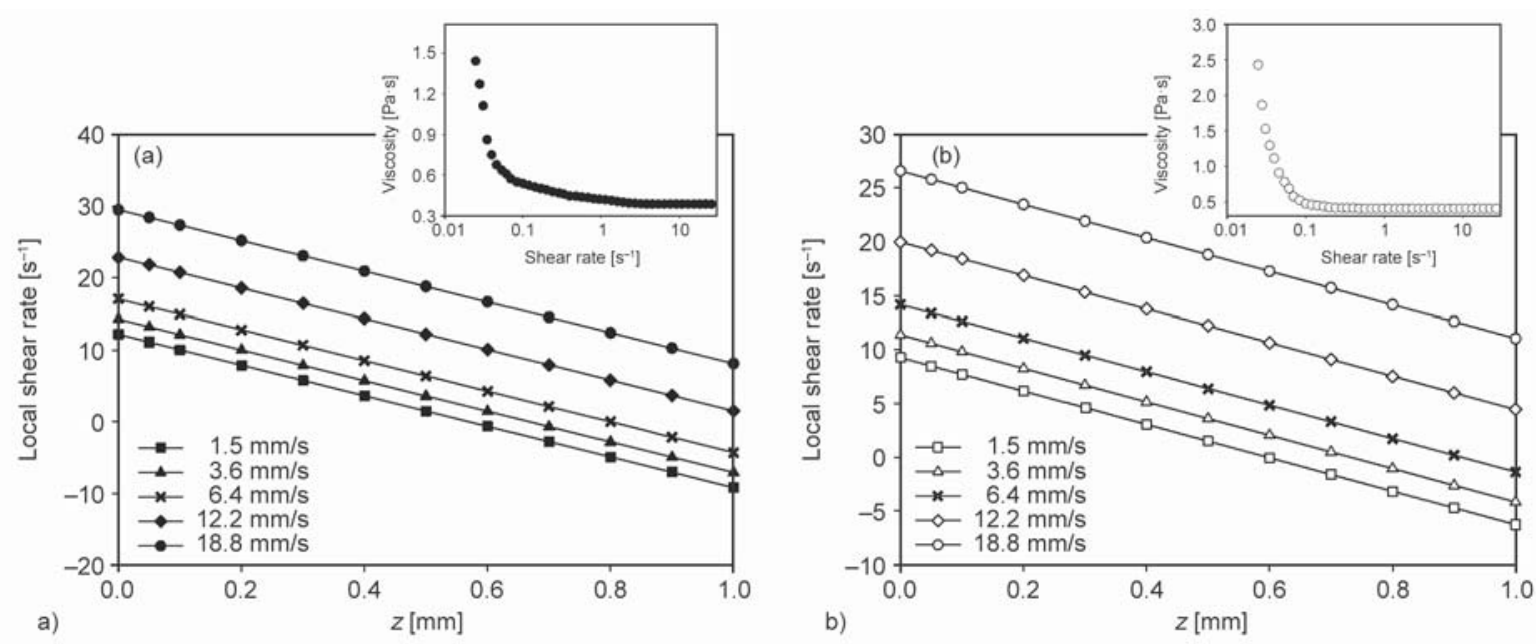

Figure 6. The shear rate profiles underneath the casting blade at several draw velocities for (a) PI-DDM and (b) PI-6FADE solutions. The inset graphs represent the semi-logarithmic plots of the solution viscosity (at $17 \mathrm{~g} / \mathrm{dl}$ ) against the shear rates beneath the blade. 
Chou et al. [32] developed a semi-empirical formula, which relate the layer thickness to solution viscosity as Equation (3):

$$
t_{\mathrm{dry}}=0.5 \cdot \alpha \cdot \beta \cdot \frac{\rho_{\mathrm{f}}}{\rho_{\mathrm{s}}} \cdot h \cdot\left[1+\left(h^{2} \Delta P\right) \cdot(6 \eta U L)^{-1}\right]
$$

where $t_{\text {dry }}$ is the dry film thickness, $\eta$ is the solution viscosity, ${ }_{U}$ is the blade speed, ${ }_{L}$ is the length of the blade, $\rho_{\mathrm{s}}$ is the fluid density, $\rho_{\mathrm{f}}$ is the solid layer density, $\Delta P$ is the hydraulic pressure, while $\alpha$ and $\beta$ are the correction factors for side flow and weight loss during drying.

The first term from Equation (3) depicts the sheardriven flow, whereas the second one presents the pressure-driven flow. The estimated layer thickness depends on the height of the blade's edge, but also on coating speed and rheological properties of the polymer solution. During tape casting, the wet layer is sheared owing to the surface tension of the solution. Thus, the variation of the solution viscosity with the coating speed is very important. The dried film thickness is influenced by the solid content and the densification behavior of the solution. Figure 7 shows the effect of the casting speed on the PI film thickness. It was noticed that the increase casting speed determines the decreasing of the sample thickness. As supported by literature [31,32], the drag force is enhanced at higher velocities and it becomes dominant in regard with pressure force. This leads to the stretching of the solution over the peeling belt. Based on these aspects, it can be observed that, at casting speeds higher than $6 \mathrm{~mm} / \mathrm{s}$, the shear-driven flow impacts more the film thickness. In this velocity interval the thickness of the sample is lower than $255 \mu \mathrm{m}$. At the highest speed, the polymer film thickness reaches 188 and $195 \mu \mathrm{m}$ for PI-DDM and PI-6FADE, respectively. The slightly higher viscosity of the fluorinated PI solution determines relatively higher film thickness. From the described analyses, the optimal processing conditions of the PI solutions into films by tape casting were found: concentration of at $17 \mathrm{~g} / \mathrm{dl}$, shear rate range of $0-30 \mathrm{~s}^{-1}$ and casting speed of

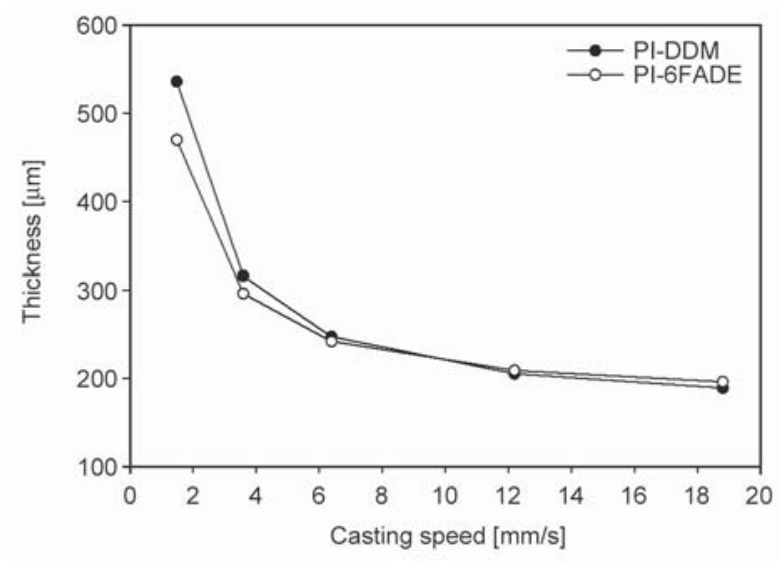

Figure 7. The estimated thickness of the PI tapes as a function of the casting speed.

$18.8 \mathrm{~mm} / \mathrm{s}$. The thickness of the dried films was measured with a digital micrometer, resulting 80.24 and $83.61 \mu \mathrm{m}$ for PI-DDM and PI-6FADE, respectively. The difference between the theoretical and experimental data could be linked to a set of cumulative factors related to film casting and drying. Perhaps the used formula (3) should also account for surface tension acting at the polymer solution/substrate interface and its variation with drying temperature. However, considering that both theory and experimental data showed that PI-6FADE film is slightly thicker than PI-DDM means that Equation (3) is a good approach to evaluate the correlation between rheology and film processing.

\subsection{Prediction of the PI permeability}

The permeability of the PIs to small gas molecules was evaluated using the theory developed by Bicerano [33], which is mainly based on the connectivity indices. In membrane oxygenator applications, a particular attention must be given to the transport of oxygen and carbon dioxide transport. In order to ensure a proper functioning of such biomedical device, one must ensure an enhanced $\mathrm{O}_{2}$ exchange and $\mathrm{CO}_{2}$ removal. Therefore, the permeability $(P)$ of the studied PIs to these types of penetrant molecules is estimated using Equations (4) and (5):

$$
\begin{aligned}
& P\left(\mathrm{O}_{2}\right)=4991.6 \exp \left[-0.017622\left(\frac{E_{\text {coh }}}{V}-196 \frac{V}{V_{\mathrm{W}}}+110 \frac{N_{\text {rot }}}{N}-57 \frac{N_{\text {per }}}{N}\right)\right] \\
& P\left(\mathrm{CO}_{2}\right)=31077.9 \exp \left[-0.019195\left(\frac{E_{\text {coh }}}{V}-196 \frac{V}{V_{\mathrm{W}}}+110 \frac{N_{\text {rot }}}{N}-57 \frac{N_{\text {per }}}{N}\right)\right]
\end{aligned}
$$


where $E_{\text {coh }}$ is the cohesion energy, $V$ is the molar volume, $V_{\mathrm{W}}$ is the van der Waals volume, $N_{\text {rot }}$ is a factor related to the rotational degrees of freedom, $N$ quantifies the non-hydrogen atoms and $N_{\text {per }}$ is a specific structural parameter defined in [33].

The parameters used for the prediction of the PI permeability were calculated on the basis of the Bicerano

Table 3. The values of zeroth-order atomic connectivity indices $\left({ }^{0} \chi\right.$ and $\left.{ }^{0} \chi^{v}\right)$, first-order bond connectivity indices $\left({ }^{1} \chi\right.$ and $\left.{ }^{1} \chi^{v}\right)$, molar volume $(V)$, van der Waals volume $\left(V_{\mathrm{W}}\right)$, cohesion energy $\left(E_{\mathrm{coh}}\right)$, non-hydrogen atoms $(N)$, rotational degrees of freedom $\left(N_{\text {rot }}\right)$, structural parameter $\left(N_{\text {per }}\right)$ and PI permeabilities to $\mathrm{O}_{2}$ and $\mathrm{CO}_{2}$ molecules.

\begin{tabular}{|ll|c|c|}
\hline \multicolumn{2}{|c|}{ Bicerano parameters } & PI-DDM & PI-6FADE \\
\hline${ }^{0} \chi$ & 22.15 & 38.32 \\
\hline${ }^{0} \chi^{v}$ & & 17.65 & 27.44 \\
\hline${ }^{1} \chi$ & 15.42 & 25.58 \\
\hline${ }^{1} \chi^{v}$ & & 11.03 & 16.92 \\
\hline$V$ & {$\left[\mathrm{~cm}^{3} / \mathrm{mol}\right]$} & 361.22 & 562.82 \\
\hline$V_{\mathrm{W}}$ & {$\left[\mathrm{cm}^{3} / \mathrm{mol}\right]$} & 241.13 & 379.05 \\
\hline$E_{\text {coh }}$ & {$[\mathrm{J} / \mathrm{mol}]$} & $1.81 \cdot 10^{5}$ & $2.65 \cdot 10^{5}$ \\
\hline$N$ & & 32.00 & 54.00 \\
\hline$N_{\text {rot }}$ & & 4.00 & 10.00 \\
\hline$N_{\text {per }}$ & & 25.00 & 25.00 \\
\hline$P\left(\mathrm{O}_{2}\right)$ & {$[\text { Barrer }]^{*}$} & 1.322 & 1.398 \\
\hline$P\left(\mathrm{CO}_{2}\right)$ & {$[\text { Barrer }]^{*}$} & 6.229 & 6.625 \\
\hline
\end{tabular}

${ }^{*} 1$ Barret $=\frac{10 \mathrm{~cm}^{3}(\mathrm{STP}) \mathrm{cm}^{3}}{\mathrm{~cm}^{2} \cdot \mathrm{s} \cdot \mathrm{cmHg}}$, where STP means standard temper-

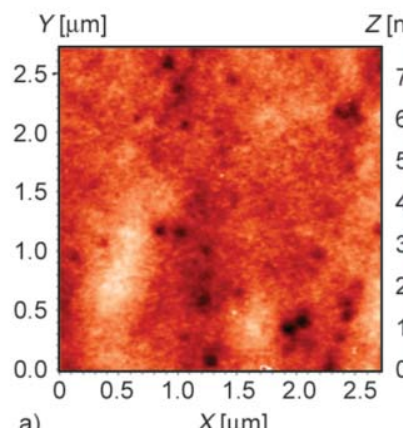

$Z[\mathrm{~nm}] Y[\mu \mathrm{m}]$
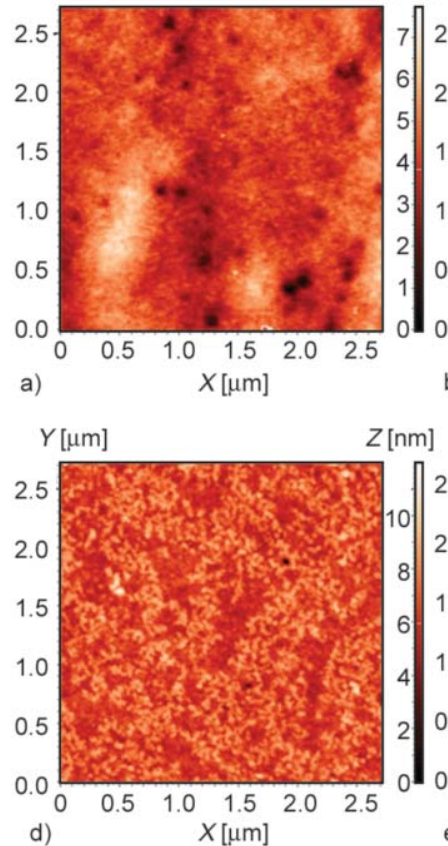

$Z$ [nm] $Y[\mu \mathrm{m}]$

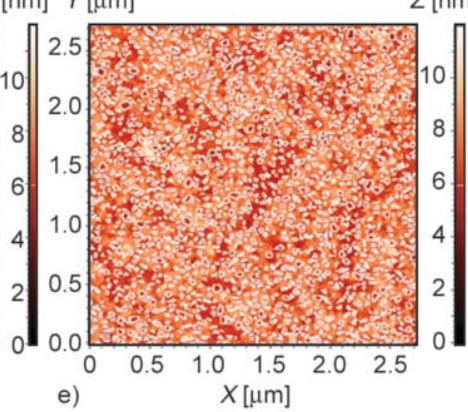

theory [33] applied to each studied polymer structure. Table 3 presents the results obtained for all Bicerano parameters, including the permeability of both PIs to the considered gases. For both samples, the values of $P\left(\mathrm{CO}_{2}\right)$ are high enough to ensure the carbon dioxide removal, whereas the values of $P\left(\mathrm{O}_{2}\right)$ correspond to the oxygen exchange demands. It can be noticed that PI-6FADE film presents higher permeability to $\mathrm{O}_{2}$ and $\mathrm{CO}_{2}$ molecules comparatively with PI-DDM sample. This could be explained by considering that the fluorinated PI has a longer, bulky and more flexible diamine segment. All these aspects are favoring a lower chain packing and implicitly a better transport of the considered molecules through this PI film.

\subsection{Film morphology}

In order to understand the results for the polymer gas permeability, the morphology of the PI films was investigated. The AFM technique was used to obtain information on sample topography at the nanometer scale. As seen in Figure 8a, 8d, the 2D-AFM images reveal that both PI films exhibit a porous morphology, which is better evidenced in the pore maps (Figure $8 \mathrm{~b}, 8 \mathrm{e})$. The type of the diamine sequence determines significant changes in surface morphology. The PI-DDM sample presents larger and less uniform pores on the surface (according to aspect ratio diagram) and their size is ranging between 50 $340 \mathrm{~nm}$. Conversely, the PI-6FADE film displays a

Figure 8. The 2D-AFM images on $2.5 \mu \mathrm{m}^{2}$, the pore maps and pore aspect ratio distribution of (a, b, c) PI-DDM film and $(d, e, f)$ PI-6FADE film. The inset graphs represent the diagram of pore diameter distribution. 
Table 4. The parameters extracted from AFM scans of the studied PI films.

\begin{tabular}{|c|c|c|}
\hline \multicolumn{1}{|c|}{ AFM parameters } & PI-DDM & PI-6FADE \\
\hline $\begin{array}{r}\text { Variation of pore dimension } \\
{[\mathrm{nm}]}\end{array}$ & $50-347$ & $25-84$ \\
\hline Variation of pore depth [nm] & $3-8$ & $4-14$ \\
\hline Average pore diameter [nm] & $149 \pm 56$ & $43 \pm 11$ \\
\hline $\begin{array}{r}\text { Average pore aspect ratio } \\
{[\mathrm{nm}]}\end{array}$ & $2.955 \pm 0.835$ & $2.415 \pm 0.584$ \\
\hline $\begin{array}{r}\text { Average pore perimeter } \\
{[\mathrm{nm}]}\end{array}$ & $754 \pm 339$ & $122 \pm 54$ \\
\hline Average pore area $\left[\mathrm{nm}^{2}\right]$ & $20 \cdot 10^{3} \pm 15.9 \cdot 10^{3}$ & $1.56 \cdot 10^{3} \pm 0.88 \cdot 10^{3}$ \\
\hline
\end{tabular}

higher number of smaller and more uniformly distributed pores on the surface. In this case, the average pore size ranges between $2-80 \mathrm{~nm}$. For a clearer picture of the studied PI morphological features, we extracted some parameters from Figure $8 \mathrm{c}, 8 \mathrm{f}$ and listed them in Table 4. The fluorinated PI presents a smaller variation of pore dimension, higher pore depth, lower pore diameter, perimeter and aspect ratio, in comparison with PI-DDM.

Carbon dioxide is a linear molecule with the length of the $\mathrm{C}=\mathrm{O}$ bond of $116 \mathrm{pm}$, thus the overall diameter size is $232 \mathrm{pm}$. The atomic radius of oxygen is about $60 \mathrm{pm}$, making the diameter twice this, one may assume that the diameter of the molecular oxygen is $120 \mathrm{pm}$. Comparing these values with the average pore diameter of both PI films, it can be noticed that
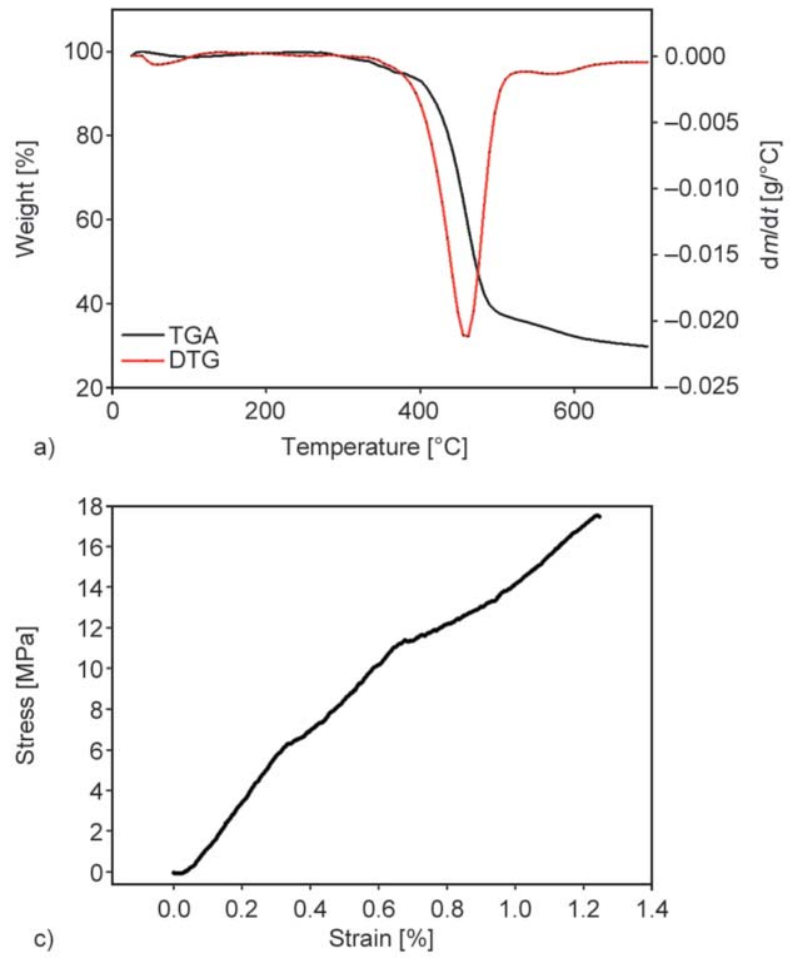

the polymer morphology facilitates the transport of the considered gas molecules, as supported by the theoretical predictions. The higher number of pores, combined with their uniform size and distribution explains the higher gas permeability of PI-6FADE.

\subsection{Thermogravimetric and tensile properties}

The results of thermal analysis of the PI films are presented in Figure 9a and 9b. Both polymers are thermally stable up to $350^{\circ} \mathrm{C}$. The main degradation characteristics are presented in Table 5. The TG and DTG graphs confirm the absence of NMP solvent and a proper drying of the samples.

The tensile properties are shown in Figure 9c and $9 d$ and Table 5 for examined films. The polymer containing DDM units has a lower elongation at break corresponding to a lower tensile strength comparatively with PI-6FADE. Also, as seen in Table 5, Young modulus is lower for the fluorinated sample. These results indicate a higher mechanical resistance for PI-6FADE film in regard with PI-DDM sample.

\subsection{Polyimide/blood interactions}

Biomedical devices, like membrane oxygenators, impose a direct contact between blood and the PI biomaterial. The interactions occurring at the bio-interface determine a series of complicated events as a result of plasma proteins adsorption and/or blood
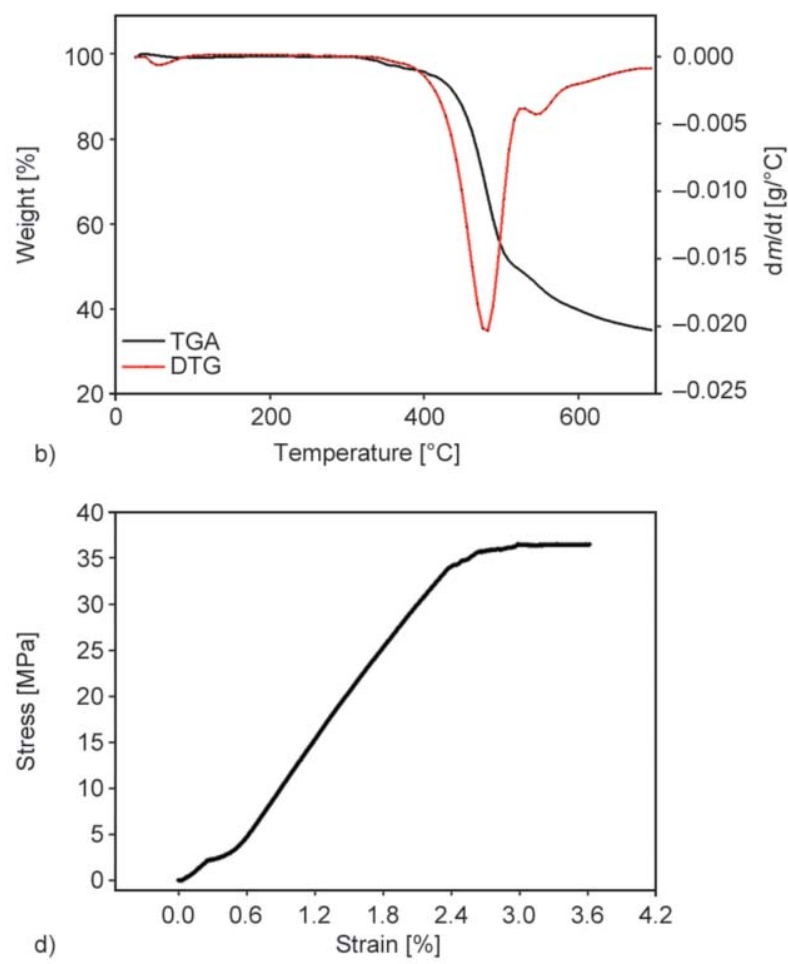

Figure 9. TGA, DTG and stress-strain graphs for (a, c) PI-DDM and (b, d) PI-6FADE samples. 
Table 5. The thermal and tensile properties of the studied PIs: glass transition temperature ( $T_{\mathrm{g}}$ - taken from reference [27]), initial decomposition temperature at which the sample displayed a 5\% weight loss (IDT), the temperature corresponding to the loss of $10 \%$ by weight $\left(T_{10}\right)$, the maximum decomposition temperature in the DTG curve $\left(T_{\mathrm{d}}\right)$, Young modulus, elongation at break $\left(\varepsilon_{\text {break }}\right)$ and tensile strength $(\sigma)$.

\begin{tabular}{|l|c|c|c|c|c|c|c|}
\hline \multicolumn{1}{|c|}{ PI code } & $\begin{array}{c}\boldsymbol{T}_{\mathbf{g}} \\
{\left[{ }^{\circ} \mathbf{C}\right]}\end{array}$ & $\begin{array}{c}\text { IDT } \\
{\left[{ }^{\circ} \mathbf{C}\right]}\end{array}$ & $\begin{array}{c}\boldsymbol{T}_{\mathbf{1 0}} \\
{\left[{ }^{\circ} \mathbf{C}\right]}\end{array}$ & $\begin{array}{c}\boldsymbol{T}_{\mathbf{d}} \\
{\left[{ }^{\circ} \mathbf{C}\right]}\end{array}$ & $\begin{array}{c}\text { Young modulus } \\
{[\mathbf{G P a}]}\end{array}$ & $\begin{array}{c}\boldsymbol{\varepsilon}_{\text {break }} \\
{[\mathbf{\%}]}\end{array}$ & $\begin{array}{c}\boldsymbol{\sigma} \\
{[\mathbf{M P A}]}\end{array}$ \\
\hline Poly(EPI-DDM) & 241 & 366 & 414 & 455 & 2.16 & 1.24 & 17.43 \\
\hline Poly(EPI-6FADE) & 215 & 407 & 441 & 481 & 1.70 & 3.61 & 36.49 \\
\hline
\end{tabular}

cells adhesion to the polymer [34]. In the first stage, the proteins are rapidly adsorbed causing their activation, which can catalyze or moderate specific biological responses to the used material. The adhesion and activation of the platelets, combined with plasma coagulation, are responsible for thrombus formation [34]. This is an undesired effect because the surface-induced thrombosis is strongly limiting the longterm reliability of the blood contacting devices.

There are several factors that dictate the material's biological responses. Among them, literature [34] highlights the importance of the surface free energy in moderating the protein and cell interactions, and finally the host response. In previous works [18-22], the hemocompatibility was assessed by calculation of the work of spreading of the blood (including its cells and proteins) on PI surface. Here, we used another approach that involves direct contact of blood with PI film surface. So, based on the contact angles of the biological fluid on EPI-based samples, the work of spreading was determined using Equation (6):

$$
\begin{aligned}
W_{\mathrm{s}} & =W_{\mathrm{a}}-W_{\mathrm{c}}=\gamma_{\mathrm{s}}-\gamma_{\mathrm{sb}}-\gamma_{\mathrm{b}}= \\
& =\left[\left(1+\cos \theta_{\mathrm{pb}}\right) \gamma_{\mathrm{b}}\right]-2 \gamma_{\mathrm{b}}
\end{aligned}
$$

where $W_{\mathrm{s}}$ is the work of spreading, $W_{\mathrm{a}}$ is the work of adhesion, $W_{\mathrm{c}}$ is the work of cohesion, $\theta_{\mathrm{pb}}$ is the contact angle of the blood on the polymer surface, $\gamma_{\mathrm{sb}}$ is the surface tension at PI/blood interface, $\gamma_{\mathrm{b}}$ and $\gamma_{s}$ are the surface tension of the blood (or its components) and PI film, respectively.

In Figure 10, one may notice the images of the blood drops on the samples and contact angle pictures for both PI films. By introducing the values of the blood

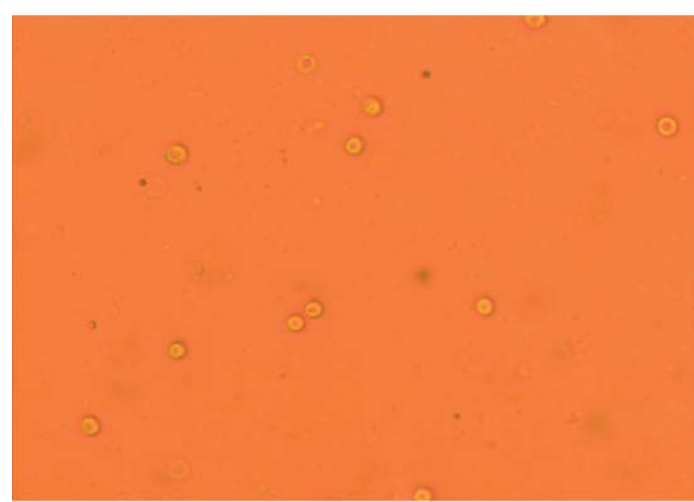

a)

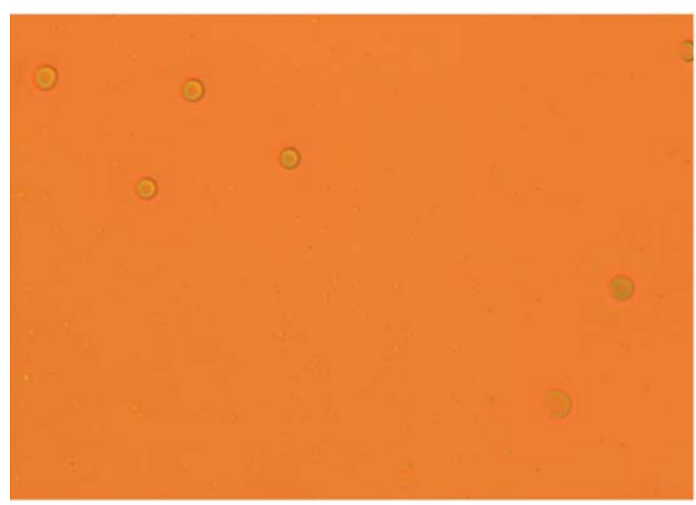

d)

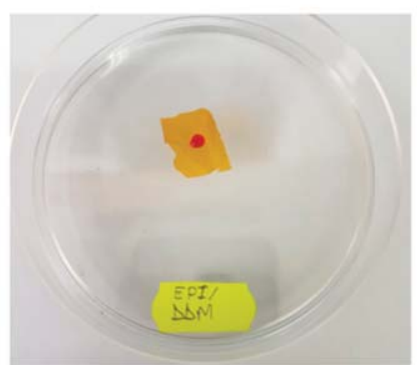

b)

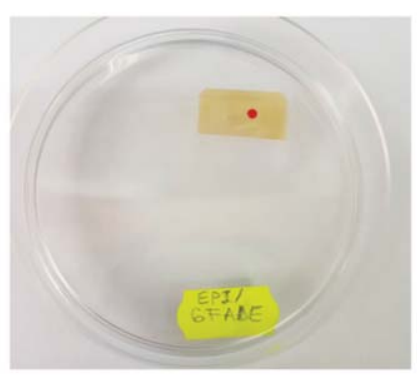

e)

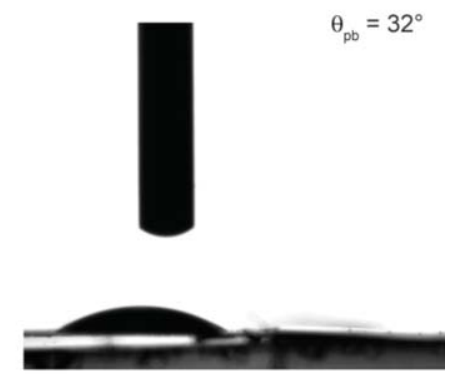

c)

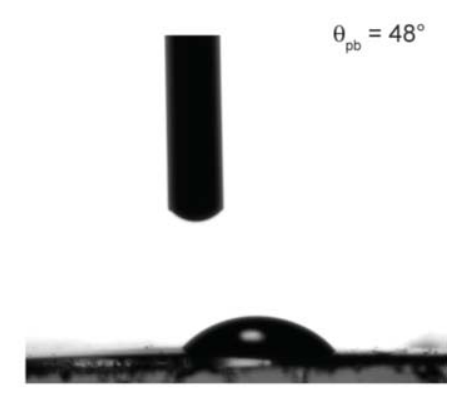

f)

Figure 10. Optical microscopy images of the PI films after interacting for 4 hours with blood, the images of the contact angle of blood drops on the samples and contact angle pictures for $(a, b, c)$ PI-DDM and (d, e, f) PI-6FADE. 
contact angles in Equation (6) and the surface energy of the biological fluid [35], the work of spreading was evaluated. According to Table 6 , this parameter takes negative values, meaning that the cohesion forces at the PI/blood interface overcome the adhesion ones. The blood fluid has low tendency to adhere on both samples, especially on PI-6FADE.

In order to obtain deeper knowledge on the bio-interactions, the balance between adhesion and cohesion forces at PI/blood components was further estimated. This was done based on the values of surface tension of each material and the surface tension at the interface. The surface tension values of the studied PI films were previously published [27] and the values are presented in Table 6 . The surface tension of the blood components were taken from literature [36-40].

For membrane oxygenator purposes, it is desirable that the surface energy features of the PIs should not

Table 6. The contact angle of blood fluid on studied PI films and the values for work of spreading with blood and its components.

\begin{tabular}{|c|c|c|}
\hline & PI-DDM & PI-6FADE \\
\hline$\theta_{\mathrm{pb}}$ & 32.00 & 48.00 \\
\hline$[\mathrm{mN} / \mathrm{m}]$ & $45.73^{*}$ & $45.43^{*}$ \\
\hline \multicolumn{3}{|c|}{$\gamma_{\mathrm{sb}}[\mathrm{mN} / \mathrm{m}]$} \\
\hline Fibrinogen & 1.79 & 0.50 \\
\hline IgG & 0.76 & 2.31 \\
\hline Albumin & 7.74 & 11.87 \\
\hline $\operatorname{IgA}$ & 11.36 & 8.19 \\
\hline Fibrin & 1.99 & 0.56 \\
\hline Lipoprotein A & 0.83 & 0.19 \\
\hline$\alpha 2$-Macroglobulin & 17.14 & 12.18 \\
\hline $\mathrm{RBC}$ & 4.52 & 2.35 \\
\hline Lymphocytes & 11.25 & 8.03 \\
\hline Monocytes & 29.36 & 31.65 \\
\hline Platelets & 17.56 & 17.09 \\
\hline \multicolumn{3}{|c|}{$W_{\mathrm{s}}[\mathrm{mN} / \mathrm{m}]$} \\
\hline Blood & -7.22 & -15.71 \\
\hline Fibrinogen & 2.44 & 3.43 \\
\hline IgG & -6.33 & -8.18 \\
\hline Albumin & -24.51 & -28.94 \\
\hline $\operatorname{IgA}$ & 7.57 & 10.43 \\
\hline Fibrin & -0.26 & 0.87 \\
\hline Lipoprotein A & 4.78 & 5.13 \\
\hline a2-Macroglobulin & -42.31 & -37.65 \\
\hline $\mathrm{RBC}$ & 4.66 & 6.52 \\
\hline Lymphocytes & 6.78 & 9.70 \\
\hline Monocytes & 10.35 & 7.76 \\
\hline Platelets & -90.07 & -89.91 \\
\hline
\end{tabular}

*taken from [27] induce significant surface adhesion of blood cells or proteins. In the first stage, the spreading of erythrocytes (RBC), lymphocytes, monocytes and platelets was determined. The data from Table 6 indicate that the PI substrates generate a low surface adhesion to these blood cells given the positive, but small values of the $W_{\mathrm{s}}$. The main role of platelets is to react to bleeding from blood vessel injury, so they form clots. In this case, the high and negative of $W_{\mathrm{s}}$ reveal that the predominant component of thrombocytes spreading on PI surface is given by cohesion forces. The RBCs represent almost $50 \%$ of blood volume and they are responsible for oxygen delivery to the tissues. They also have the high cohesion to the sample surface. The lymphocytes and monocytes are white blood cells (WBCs), which are related to the immune system. Both PIs exhibit a relatively diminished adhesion to these WBCs. Analyzing the obtained data, it can be stated that the studied imidic polymers present a low level of adhesion forces at the bio-interface with blood cells, not exceeding $10.35 \mathrm{mN} / \mathrm{m}$.

In the second stage, the hemocompatibility was discussed in terms of blood proteins spreading on the investigated PIs. Several types of proteins were selected to check their bio-interactions with our polymers, namely (1) albumin that regulates the osmotic pressure of plasma, (2) globulins (IgA and $\operatorname{IgG}$ ) that includes antibodies and participates in immune system, (3) fibrinogen and fibrin that are responsible for blood coagulation, (4) $\alpha 2$-macroglobulin may act as a carrier protein and inhibits coagulation by inhibiting thrombin, and (5) lipoprotein A contributes to the process of atherogenesis. Positive values of $W_{\mathrm{s}}$ were obtained when PI films interact with fibrinogen, IgA and lipoprotein A. This is indicative of the fact that the adhesion forces are dominant at the interface. However, given the small values of the work of spreading, it could be stated that the level of adhesion of these proteins to PI foils (i.e. below $10 \mathrm{mN} / \mathrm{m}$ ) is low. Stronger cohesion forces were noticed at both samples bio-interface with IgG, albumin, fibrin and $\alpha 2$-macroglobulin.

In the third stage, the PI blood compatibility was evaluated by placing blood (containing $3.8 \%$ anticoagulant) on the samples. After 4 hours of incubation the biological fluid was rinsed and the morphology of PI films was examined to check if blood cells adhere and thrombus appear. As remarked in Figures 10a and $10 \mathrm{~d}$, the surface characteristics of the analyzed PI films do not induce platelets aggregation, RBC 
lysis or blood clotting. Also, as displayed in optical microscopy images, the contact with PI supports do not affect the morphology of the blood cells. This aspect is a good indicatiion of a hemocompatible material [41]. More detailed investigations on these PIs from the medical point of view will be the subject of a future work.

\section{Conclusions}

Two semi-alicyclic PIs were prepared by thermal imidization and characterized for membrane oxygenator purposes. The higher chain flexibility of PI6FADE causes a more pronounced shear thinning behavior of the corresponding solutions in NMP comparatively with PI-DDM, which is useful for tape casting processing. For both samples, the pseudoplastic behavior is maintained at concentrations in the semi-dilute entangled regime. Numerical simulations were performed to determine the velocity and shear rate profiles under the casting blade. The data were transferred in the rheological curves and it was observed that viscosity still ranges with the shear rate. Proper films are obtained at a viscosity corresponding to $17 \mathrm{~g} / \mathrm{dl}$ concentration in entangled regime, shear rate between $0-30 \mathrm{~s}^{-1}$ and a casting speed of $18.8 \mathrm{~mm} / \mathrm{s}$. The $\mathrm{O}_{2}$ and $\mathrm{CO}_{2}$ permeability of PI-DDM was predicted to be lower in regard with that of PI6FADE. The AFM data explains this theoretical result by revealing that $6 \mathrm{FADE}$ segment renders large number of small pores, that are uniformly distributed on the PI film surface favoring a higher permeability, which is useful for membrane oxygenator purposes. Hemocompatibility tests relied on measuring the adhesion of blood drops on PI samples and monitoring blood cell adhesion by optical microscopy. The results indicated no prevalent adhesion of cells or plasma proteins and no thrombus formation, confirming the biocompatibility of the PI samples. It can be concluded that the higher flexibility and hydrophobicity of the PI-6FADE film renders higher tensile strength and better hemocompatibility in regard with PI-DDM sample. These properties recommend PI-6FADE as a potential candidate for membrane oxygenator applications.

\section{Acknowledgements}

This work is dedicated to Dr. Silvia Ioan - Senior Researcher from 'Petru Poni' Institute of Macromolecular Chemistry. The financial support of European Social Fund for Regional Development, Competitiveness Operational Programme Axis 1 - Project 'Petru Poni Institute of Macromolecular Chemistry - Interdisciplinary Pol for Smart Specialization through Research and Innovation and Technology Transfer in Bio (nano)polymeric Materials and (Eco)Technology', InoMatPol (ID P_36_570, Contract 142/10.10.2016, cod MySMIS: $107464)$ is gratefully acknowledged.

\section{References}

[1] Kulinets I.: Biomaterials and their applications in medicine. in 'Regulatory affairs for biomaterials and medical devices' (eds.: Amato S. F., Ezzell Jr R. M.) Woodhead, Amsterdam, 1-10 (2015).

https://doi.org/10.1533/9780857099204.1

[2] Amoako K., Gbyli R.: Improving the hemocompatibility of biomedical polymers. in 'Hemocompatibility of biomaterials for clinical applications blood-biomaterials interactions' (ed.: Siedlecki C. A.) Woodhead, Oxford, 223-252 (2018).

https://doi.org/10.1016/B978-0-08-100497-5.00016-1

[3] Ekdahl K. N., Hong J., Hamad O. A., Larsson R., Nilsson B.: Evaluation of the blood compatibility of materials, cells, and tissues: Basic concepts, test models, and practical guidelines. in 'Complement therapeutics. Advances in experimental medicine and biology' (eds.: Lambris J., Holers V., Ricklin D.) Springer, Boston, Vol 735, 257-270 (2013).

[4] Gorbet M. B., Sefton M. V.: Biomaterial-associated thrombosis: Roles of coagulation factors, complement, platelets and leukocytes. Biomaterials, 25, 5681-5703 (2004).

https://doi.org/10.1016/j.biomaterials.2004.01.023

[5] Wang J., Pan C. J., Huang N., Sun H., Yang P., Leng Y. X., Chen J. Y., Wan G. J., Chu P. K.: Surface characterization and blood compatibility of poly(ethylene terephthalate) modified by plasma surface grafting. Surface and Coatings Technology, 196, 307-311 (2005).

https://doi.org/10.1016/j.surfcoat.2004.08.161

[6] Yang M-C., Lin W-C.: Surface modification and blood compatibility of polyacrylonitrile membrane with immobilized chitosan-heparin conjugate. Journal of Polymer Research, 9, 201-206 (2002).

https://doi.org/10.1023/A:1021347810130

[7] Grombe R., Gouzy M. F., Freudenberg U., Pompe T., Zschoche S., Simon F., Eichhorn K-J., Janke A., Voit B., Werner C.: Sulfated cellulose thin films with antithrombin affinity. Express Polymer Letters, 3, 733-742 (2009).

https://doi.org/10.3144/expresspolymlett.2009.91 
[8] Khorasani M. T., Mirzadeh H.: In vitro blood compatibility of modified PDMS surfaces as superhydrophobic and superhydrophilic materials. Journal of Applied Polymer Science, 91, 2042-2047 (2004).

https://doi.org/10.1002/app.13355

[9] Yuan J., Lin S., Shen J.: Enhanced blood compatibility of polyurethane functionalized with sulfobetaine. Colloids and Surfaces B: Biointerfaces, 66, 90-95 (2008). https://doi.org/10.1016/j.colsurfb.2008.05.020

[10] Richardson Jr. R. R., Miller J. A., Reichert W. M.: Polyimides as biomaterials: Preliminary biocompatibility testing. Biomaterials, 14, 627-635 (1993).

https://doi.org/10.1016/0142-9612(93)90183-3

[11] Georgiev A., Dimov D., Spassova E., Assa J., Dineff P., Danev G.: Chemical and physical properties of polyimides: Biomedical and engineering applications. in 'High performance polymers - Polyimides based' (ed.: Abadie J. M.) Intech, Rijeka, 65-84 (2012). https://doi.org/10.5772/53918

[12] Haggerty H. S., Lusted H. S.: Histological reaction to polyimide films in the cochlea. Acta Oto-Laryngologica, 107, 13-22 (1989). https://doi.org/10.3109/00016488909127474

[13] Seo J-M., Kim S. J., Chung H., Kim E. T., Yu H. G., Yu S. K.: Biocompatibility of polyimide microelectrode array for retinal stimulation. Materials Science and Engineering C, 24, 185-189 (2004).

https://doi.org/10.1016/j.msec.2003.09.019

[14] Kawakami H., Nagaoka S., Kubota S.: Gas transfer and in vitro and in vivo blood compatibility of a fluorinated polyimide membrane with an ultrathin skin layer. ASAIO Journal, 42, M871-M876 (1996). https://doi.org/10.1097/00002480-199609000-00116

[15] Niwa M., Kawakami H., Kanno M., Nagaoka S., Kanamori T., Shinbo T., Kubota S.: Gas transfer and blood compatibility of asymmetric polyimide hollow fiber. Journal of Biomaterials Science, Polymer Edition, 12, 533-542 (2001). https://doi.org/10.1163/156856201300194261

[16] Nagaoka S., Kanno M., Kawakami H., Kubota S.: Evaluation of blood compatibility of fluorinated polyimide by immunolabeling assay. Journal of Artificial Organs, 4, 107-112 (2001). https://doi.org/10.1007/BF02481420

[17] Niwa M., Kawakami H., Nagaoka S., Kanamori T., Morisaku K., Shinbo T., Matsuda T., Sakai K., Kubota S.: Development of a novel polyimide hollow-fiber oxygenator. Artificial Organs, 28, 487-495 (2004). https://doi.org/10.1111/j.1525-1594.2004.07257.x

[18] Popovici D., Barzic A. I., Stoica I., Butnaru M., Ioanid G. E., Vlad S., Hulubei C., Bruma M.: Plasma modification of surface wettability and morphology for optimization of the interactions involved in blood constituents spreading on some novel copolyimide films. Plasma Chemistry and Plasma Processing, 32, 781-799 (2012).

https://doi.org/10.1007/s11090-012-9376-x
[19] Nica S-L., Hulubei C., Stoica I., Ioanid G. E., Ioan S.: Surface properties and blood compatibility of some aliphatic/aromatic polyimide blends. Polymer and Engineering Science, 53, 263-272 (2013). https://doi.org/10.1002/pen.23260

[20] Stoica I., Barzic A. I., Hulubei C.: Surface wettability and morphology implications on semi-alicyclic polyimide hemocompatibility. in '2015 E-Health and Bioengineering Conference (EHB), Iasi, Romania’ 1-4 (2015). https://doi.org/10.1109/EHB.2015.7391499

[21] Buruiana L. I., Barzic A. I., Stoica I., Hulubei C.: Evaluation of blood cells and proteins spreading on imidic polymers containing alicyclic sequences. Journal of Polymer Research, 23, 1-8 (2016). https://doi.org/10.1007/s10965-016-1110-6

[22] Popovici D., Vlad S., Stoica I., Vasilescu D. S.: Evaluation of bio- and hemo-compatibility of two partially aliphatic copolyimides modified by plasma and chemical treatment. University Politehnica of Bucharest Scientific Bulletin Series B, 78, 149-160 (2016).

[23] Dickens S. H.: Polymerizable conditioners for adhesive bonding to dentin and enamel. U.S. Patent 09327180 , USA (1999).

[24] Stoica I., Barzic A. I., Butnaru M., Doroftei F., Hulubei C.: Surface topography effect on fibroblasts population on epiclon-based polyimide films. Journal of Adhesion Science and Technology, 29, 2190-2207 (2015). https://doi.org/10.1080/01694243.2015.1060061

[25] Berni A., Mennig M., Schmidt H.: Doctor blade. in 'Solgel technologies for glass producers and users' (eds.: Aegerter M. A., Mennig M.) Springer, Boston, 89-92 (2004).

https://doi.org/10.1007/978-0-387-88953-5 10

[26] Hulubei C., Popovici D.: Novel polyimides containing alicyclic units. Synthesis and characterization. Revue Roumaine de Chimie, 56, 209-215 (2011).

[27] Barzic A. I., Hulubei C., Stoica I., Albu R. M.: Insights on light dispersion in semi-alicyclic polyimide alignment layers to reduce optical losses in display devices. Macromolecular Materials and Engineering, in press (2019). https://doi.org/10.1002/mame.201800235

[28] Bian Y., Li P., Zhao N.: Effects of solvent quality on contact formation dynamics of polymer chain. Chemical Physics Letters, 703, 63-71 (2018). https://doi.org/10.1016/j.cplett.2018.04.009

[29] Krause W. E., Bellomo E. G., Colby R. H.: Rheology of Sodium Hyaluronate under physiological conditions. Biomacromolecules, 2, 65-69 (2001). https://doi.org/10.1021/bm0055798

[30] Sisko A. W.: The flow of lubricating greases. Industrial and Engineering Chemistry, 50, 1789-1792 (1958). https://doi.org/10.1021/ie50588a042 
[31] Jabbari M., Bulatova R., Hattel J. H., Bahl C. R. H.: Quasi-steady state power law model for flow of $\left(\mathrm{La}_{0.85} \mathrm{Sr}_{0.15}\right)_{0.9} \mathrm{MnO}_{3}$ ceramic slurry in tape casting. Materials Science and Technology, 29, 1080-1087 (2013). https://doi.org/10.1179/1743284713Y.0000000255

[32] Chou Y. T., Ko Y. T., Yan M. F.: Fluid flow model for ceramic tape casting. Journal of American Ceramic Society, 70, C280-C282 (1987).

https://doi.org/10.1111/j.1151-2916.1987.tb04900.x

[33] Bicerano J.: Prediction of polymer properties. Marcel Dekker, New York (2002).

[34] Xu L-C., Bauer J. W., Siedlecki C. A.: Proteins, platelets, and blood coagulation at biomaterial interfaces. Colloids and Surfaces Part B, 124, 49-68 (2014). https://doi.org/10.1016/j.colsurfb.2014.09.040

[35] Agathopoulos S., Nikolopoulos P.: Wettability and interfacial interactions in bioceramic-body-liquid systems. Journal of Biomedical Materials Research, 29, 421-429 (1995).

https://doi.org/10.1002/jbm.820290402

[36] van Oss C. J., Absolom D. R., Neumann A. W., Zingg W.: Determination of the surface tension of proteins I. Surface tension of native serum proteins in aqueous media. Biochimica et Biophysica Acta (BBA) - Protein Structure, 670, 64-73 (1981).

https://doi.org/10.1016/0005-2795(81)90049-0
[37] van Oss C. J.: Surface properties of fibrinogen and fibrin. Journal of Protein Chemistry, 9, 487-491 (1990). https://doi.org/10.1007/BF01024625

[38] van Oss C. J.: Long-range and short-range mechanisms of hydrophobic attraction and hydrophilic repulsion in specific and aspecific interactions. Journal of Molecular Recognition, 16, 177-190 (2003).

https://doi.org/10.1002/jmr.618

[39] Vijayanand K., Deepak K., Pattanayak D. K., Rama Mohan T. R., Banerjee R.: Interpenetring blood-biomaterial interactions from surface free energy and work of adhesion. Trends in Biomaterials and Artificial Organs, 18, 73-83 (2005).

[40] Aken W.: Interaction of blood cells with materials: Red cells and leucocytes. in 'Polymers: Their properties and blood compatibility' (ed.: Dawids S.) Kluwer Academic Publishers, London, 13-19 (1989).

[41] Sharma C. P.: Blood-compatible materials: A perspective. Journal of Biomaterials Applications, 15, 359-381 (2001). https://doi.org/10.1106/YY8L-M4DD-AGW1-QAGU 